\title{
Feature Extraction and Application of Bio-Signal
}

\author{
Iswanto Iswanto, Bakhtiar Abbas, V. Gomathy, K. Shankar, Phong Thanh Nguyen, Wahidah \\ Hashim, Andino Maseleno
}

\begin{abstract}
Biosignals have turned into a significant pointer for medical diagnosis and consequent treatment, yet in addition uninvolved health monitoring. Extracting important highlights from biosignals can help individuals comprehend the human useful state, with the goal that up and coming unsafe side effects or disease can be lightened or stayed away from. There are two fundamental methodologies ordinarily used to get valuable highlights from biosignals, which are hand-engineering and deep learning. Most of the examination in this field centers around hand- engineering highlights, which require space explicit specialists to structure calculations to remove important highlights. In the most recent years, a few investigations have utilized profound figuring out how to biologically take in highlights from crude biosignals to make include extraction calculations less reliant on people. Biosignals give correspondence among biosystems and are our essential wellspring of data on their conduct. Translation and change of signal are significant subjects of this content. Biosignals, similar to all signal, must be conveyed by some type of vitality. Biosignals can be estimated straightforwardly from their biological source, however frequently outer vitality is utilized to gauge the cooperation between the physiological framework and outside vitality. Estimating a biosignal involves changing over it to an electric signal utilizing a device known as a biotransducer. The resultant analog signal is frequently changed over to an advanced (discrete-time) signal for preparing in a PC. These investigations have likewise shown promising outcomes in an assortment of biosignal applications. In this overview, we audit various kinds of biosignals and the principle ways to deal with concentrate highlights from the signal with regards to biomedical applications.
\end{abstract}

Keywords : Biosignals, EEG, BCI, ECG, EMG.

\section{INTRODUCTION}

During the most recent decades, data about the emotional condition of clients has turned out to be increasingly more significant in PC based advancements. Biosignals have transformed into a noteworthy pointer for restorative determination and ensuing treatment, yet what's more uninvolved wellbeing checking. Removing significant features from biosignals can enable people to fathom the human helpful state, with the objective that best in class risky symptoms or sickness can be helped or avoided. There are two basic procedures normally used to get important features from biosignals, which are hand-designing and profound learning. The majority of the assessment in this field revolves around hand-designing features, which require space express pros to structure estimations to evacuate significant features. In the latest years, a couple of examinations have used significant making sense of how to naturally take in features from rough biosignals to make incorporate extraction estimations less dependent on individuals. Biosignals give correspondence among biosystems and are our basic wellspring of information on their direct. Interpretation and change of sign are noteworthy subjects of this substance. Biosignals, like all sign, must be passed on by some kind of imperativeness. Biosignals can be assessed clearly from their natural source, anyway as often as possible external imperativeness is used to check the collaboration between the physiological structure and outside essentialness. Evaluating a biosignal includes changing over it to an electric sign using a gadget known as a biotransducer. The resultant simple sign is habitually changed over to a progressed (discrete-time) signal for getting ready in a PC. These examinations have in like manner demonstrated promising results in a collection of biosignal applications. In this review, we review different sorts of biosignals and the standard approaches to manage concentrate features from the sign with respect to biomedical applications.

Revised Manuscript Received on July 22, 2019

* Correspondence Author

Iswanto Iswanto, Department of Engineer Profession Program, Universitas Muhammadiyah Yogyakarta, Yogyakarta, Indonesia. E-mail: iswanto_te@umy.ac.id

Bakhtiar Abbas, Sekolah Tinggi Ilmu Ekonomi Enam Enam, Indonesia

V. Gomathy, Associate Professor, Department of EEE, Sri Krishna College of Engineering and Technology, Coimbatore. E-Mail: gomathyv@ @skcet.ac.in

K. Shankar, Department of Computer Applications, Alagappa University, Karaikudi, India. E-mail: shankarcrypto@gmail.com

Phong Thanh Nguyen*, Department of Project Management, Ho Chi Minh City Open University, Vietnam. E-mail: phong.nt@ou.edu.vn

Wahidah Hashim, Institute of Informatics and Computing Energy, Universiti Tenaga Nasional, Malaysia.

Andino Maseleno, Institute of Informatics and Computing Energy, Universiti Tenaga Nasional, Malaysia. 


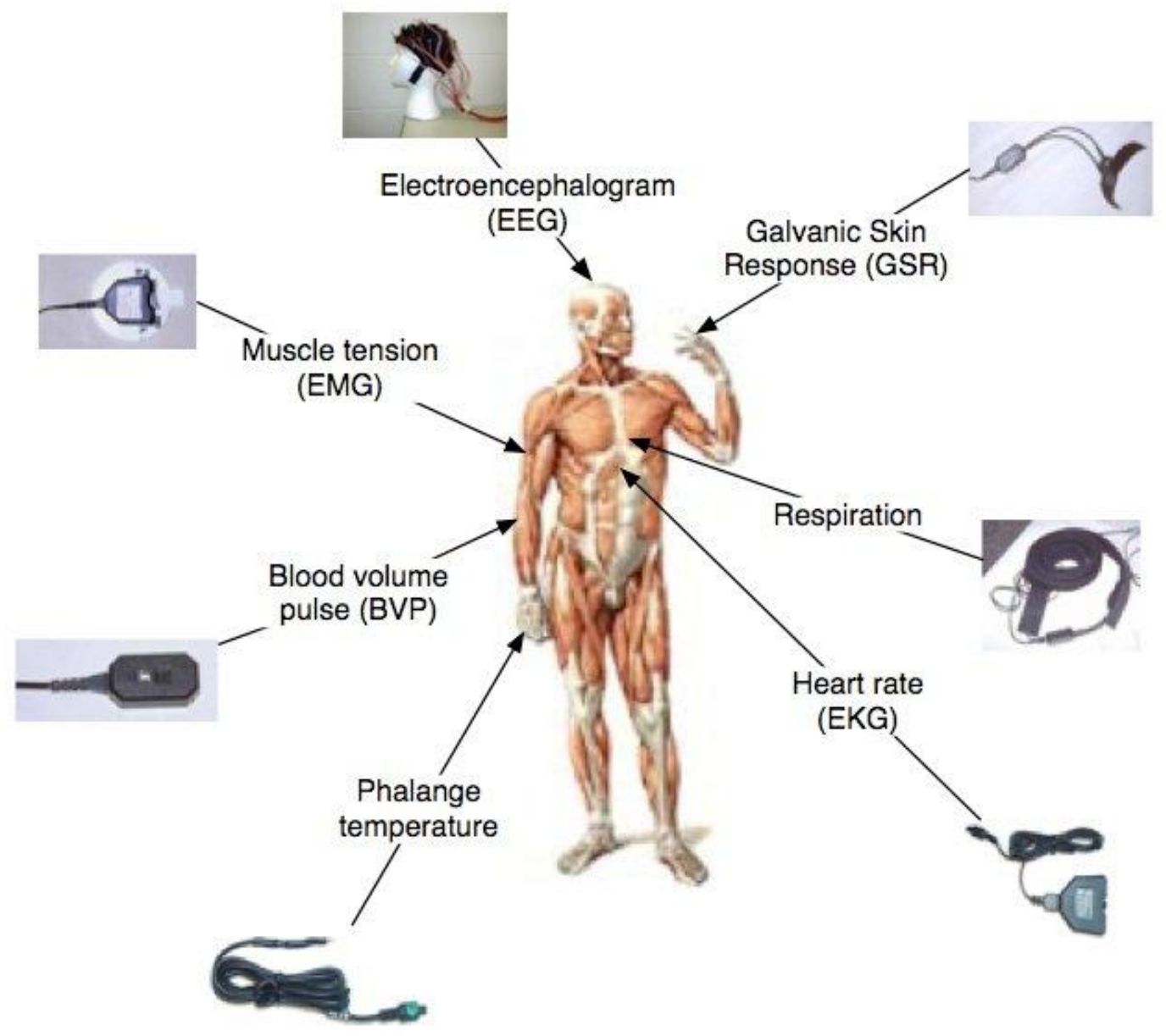

Figure 1: Examples of Biosignals

Emotional recognition frameworks, managing biological sign, display exhibitions running from $40 \%$ to $90 \%$ contingent upon the number. Biosignals and the frameworks that produce them have a few significant properties: they can be stationary or non-stationary, direct or nonlinear, and deterministic or stochastic (i.e., irregular). Biosignals regularly contain noise, which is an undesirable sign segment.

\section{BIO-SIGNALS}

Following information securing, biological signal are investigated so as to recover valuable data. Essential strategies for sign investigation (e.g., amplification, filtering, digitization, processing, and storage) can be connected to numerous biological sign. These strategies are commonly cultivated with basic electronic circuits or with advanced PCs. Notwithstanding these basic techniques, refined computerized handling strategies are very normal and can essentially improve the nature of the recovered information. These incorporate sign averaging, wavelet investigation, and man-made reasoning strategies. Bio-signals checking is a medicinal mediation characterized as the represent accumulation and examination of cardiovascular, respiratory, and body temperature information, so as to decide and avert confusions (businesswire). The bio-signals esteems arranged in a range over typical qualities happen if there should be an occurrence of illnesses, and a modification of indispensable signs is utilized to assess a patient's advancement. The biomedical sign are estimated utilizing obtrusive and noninvasive sensors. The two sorts of sensors, obtrusive and noninvasive are wearable devices for health monitoring (Figure 2).

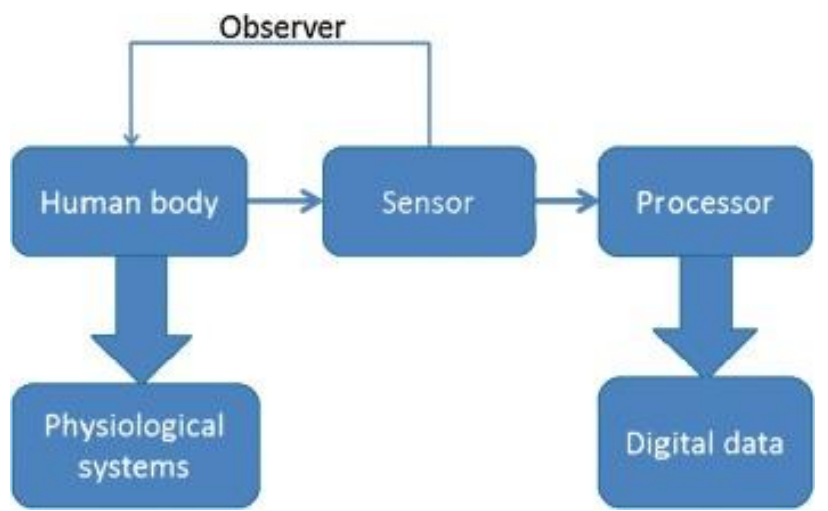

Figure 2: Flow Demonstration of research

Biological signal are the impression of collected activity possibilities of subdermal tissues of a living being. Its quality implies the ionic and electrical exercises of the strong and the neural cells in a synchronized way. Being a mosaic model of a living design, the resultant vectors of biological signal have transient just as spatial portrayal. These sign are stochastic in nature. 
Therapeutic demonstrative instruments are pervasive utilizing the help of restorative sign. Over the span of time, a lot of advancement has been accomplished in the field of therapeutic sign handling for the improvement of the signal to-noiseratio, extraction of highlights from those filtered signalsand grouping of the extricated signal for clinical applications. This extraordinary issue underscored the ongoing advancement of therapeutic signal handling, improvement of calculations, and more extensive clinical applications.

Biomedical sign and picture preparing care of includes various interests in the instructive and research field in biomedical structure. With the upgraded physiological information, a wide blend of creative works in clinical approachs utilizes this idea in the therapeutic applications. Further, prompted figurings dissected in the sign and picture preparing field typically wrapped up by time-rehash delineation approaches in the field of neurosciences, practical imaging and cardiovascular structure. In the impelled world, a part of the imaging modalities are as of now for the most part open, that can deal with crafted by disease and give definite information. The blend of electrophysiological sign and imaging techniques is a striking philosophy in the therapeutic field. Entropy-based kernal extraction system is being utilized for the investigation of the nonlinear and nonstationaryepoch signals. This sort of methodology indicates robustness in noise reduction. AI calculations are likewise being utilized for constant component extraction (design extraction) from tympanic temperature profiles. Quadratic support vector machine calculations were additionally found to improve the precision of the recognition mechanism.

\section{ELECTRICAL BIOSIGNALS}

Biosignals can be assessed clearly from their characteristic source, anyway every now and again external essentialness is used to check the collaboration between the physiological structure and outside imperativeness. Evaluating a biosignal includes changing over it to an electric signal using a device known as a biotransducer. The resultant simple sign is much of the time changed over to a progressed (discrete-time) signal for planning in a PC.

\section{Electroencephalogram (EEG)}

Of late, personality PC interface and keen sign division have pulled in an extraordinary eagerness reaching out from medication to military goals. To energize cerebrum PC interface get together, a specialist strategy for feature extraction from EEG signal is needed. Your neurotransmitters pass on by methods for electrical main thrusts and are dynamic continually, despite when you're resting.

This activity shows up as wavy lines on an EEG recording. An EEG is one of the essential investigative tests for epilepsy. An EEG can in like manner accept an occupation in diagnosing other personality issue.

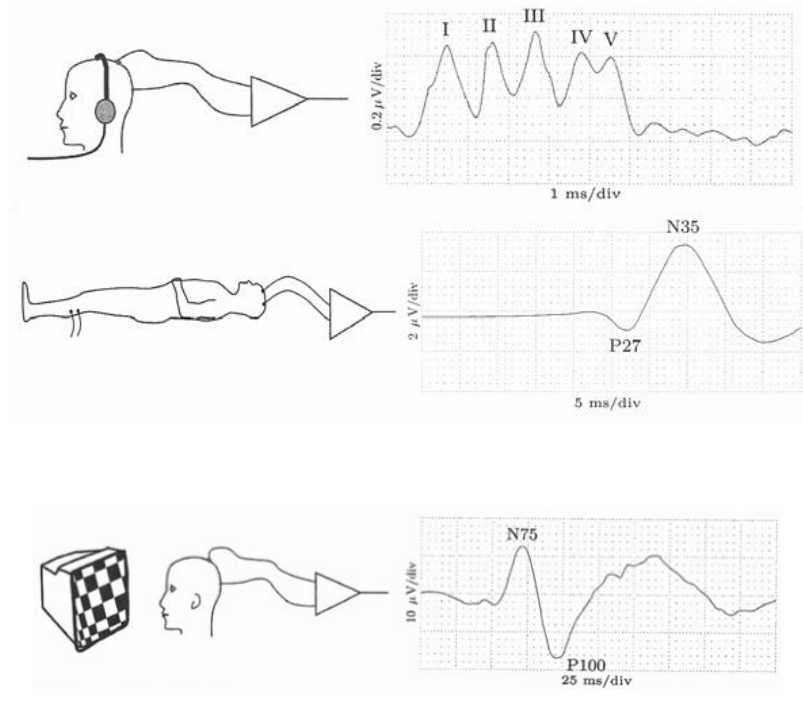

Figure 3: EEG

\section{Electrocardiogram (ECG)}

The electrocardiogram (ECG or EKG) is a demonstrative apparatus that is routinely used to evaluate the electrical and strong elements of the heart. An electrocardiogram (ECG) is a test which estimates the electrical action of your heart to indicate whether it is working typically. An electrocardiogram can be a helpful method to see if your hypertension has made any harm your heart or veins. Along these lines, you might be approached to have an ECG when you are first determined to have hypertension.

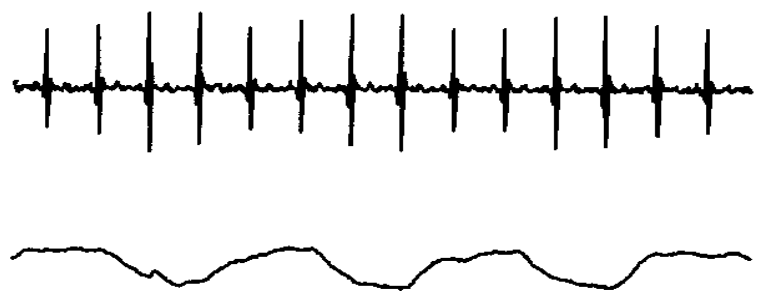

Figure 4 : ECG and Respiration

\section{Electromyogram (EMG)}

Electromyography (EMG) is andiagnostic method that evaluates the prosperity condition of muscles and the nerve cells that control them. These nerve cells are known as engine neurons. They transmit electrical sign that reason muscles to contract and loosen up. An EMG makes an elucidation of these sign into outlines or numbers, helping masters to make an examination.

A pro will for the most part orchestrate an EMG when someone is showing reactions of a muscle or nerve issue. These reactions may consolidate shuddering, deadness, or unexplained shortcoming in the members. EMG results can empower the authority to examine muscle issue, nerve issue, and disarranges affecting the relationship among nerves and muscles. 


\section{Electrooculogram (EOG)}

Electrooculogram (EOG) signal is one of the bioelectric sign picked up from the human body to consider the advancements of eyes and besides to design and make assistive devices. These devices can be flexibility devices, video gaming devices or some other assistive device.

Electrooculography (EOG) is a strategy for estimating the corneo-retinal standing potential that exists between the front and the back of the human eye. The subsequent signal is known as the electrooculogram. Essential applications are in ophthalmological analysis and in chronicle eye developments.

\section{Electrodermal Activity (EDA)}

It suggests the autonomic changes in the electrical properties of the skin. The skin conductance, which can be non-prominently evaluated by applying a low steady voltage, is one of the most by and large pondered electrodermal portions. EDA demonstrates the movements in autonomic attentive energy that are joined with energetic and mental states, as such transforms into a run of the mill extent of autonomic tangible framework development.

\section{FEATURE EXTRACTION BY UTILIZING ARTIFICIAL INTELLIGENCE}

Artificial Intelligence is a bit of ML that uses multi-layer of straight and moreover non-direct arranging units to learn bumbled of delineations that stimulate the resulting ML. There are different sorts of Artificial Intelligence intended to demonstrate various sorts of information. Around there we will portray just three instances of the most typically utilized noteworthy learning calculations in biosignal applications: stacked autoencoder, convolutional neural network and recurrent neural structure.

\section{Stacked Auto encoder (SAE)}

It is a neural structure containing different layers of autoencoders in which the yields of each layer is solicited to the devotion from the going with layer. An autoencoder is a neural system including only one verified layer. It is a solo learning calculation equipped for extricating great component introductions from an a lot of unlabeled data.By setting the target estimation of the autoencoder to be indistinct from the data, the autoencoder endeavors to get settled with a segment outline that can be used to re-attempt the data. Stacking these autoencoders, in like way, attracts the structure to take in gainful part depictions from EEG data, as the resulting layers can utilize the features snatched from the past layers to pass on a fundamental level reliably obliging features.

\section{Convolutional Neural Network (CNN)}

It is a neural system including convolutional and pooling layers. Each convolutional layer contains an enormous measure of neurons that coordinate with only a bordering locale or a fix of the data (e.g., a period window in biosignals) to perceive different models. Each neuron contain different trainable parameters, or a channel, which are used to convolve each fix a zone to think about the closeness to the model encoded on the parameters. The yield created utilizing all fix territories gather what is known as a section map. By sensibly turning convolutional and pooling layers, the framework can be set up to learn time-invariant adjacent part locators from high-dimensional data, for instance, pictures and biosignals.

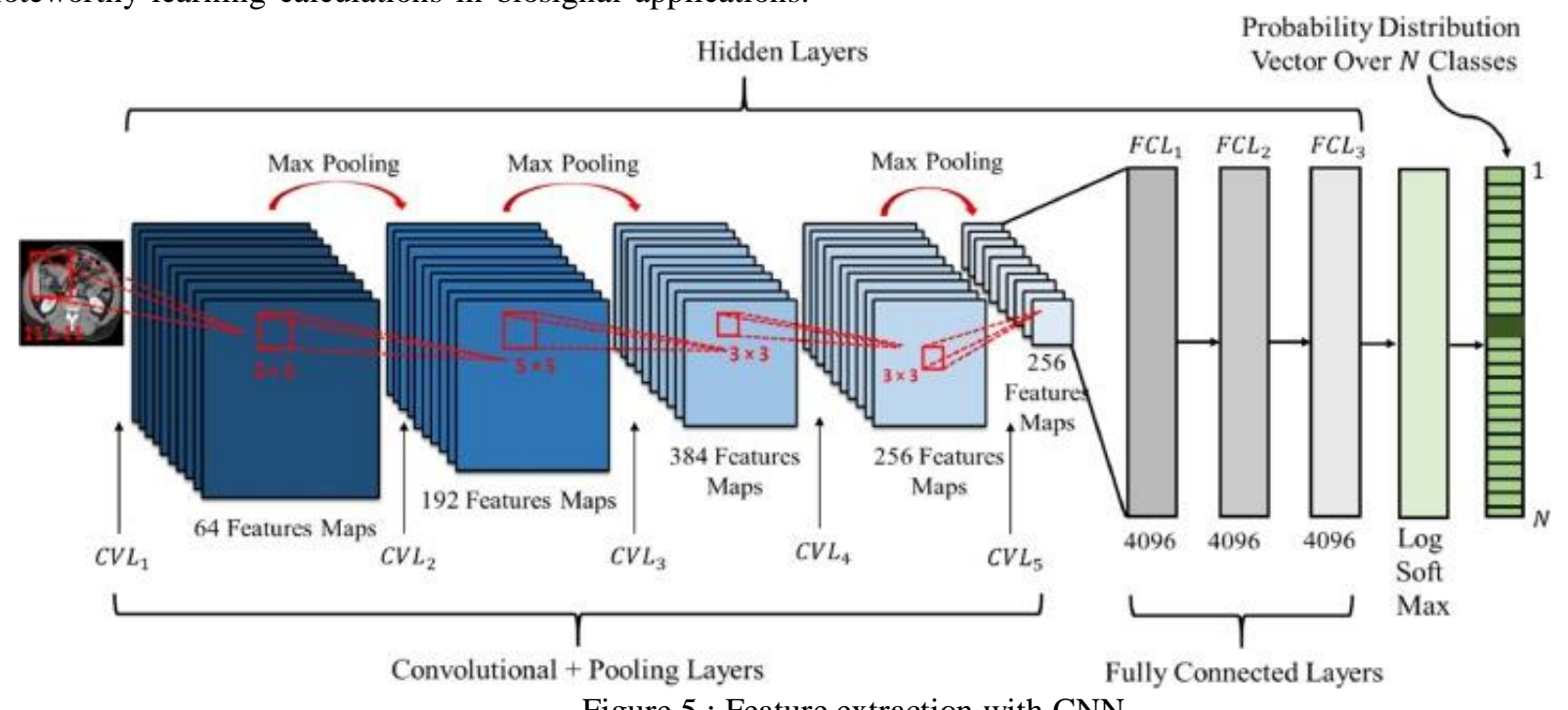

Figure 5 : Feature extraction with $\mathrm{CNN}$

\section{Redundant Neural Network (RNN)}

It is a neural framework for dealing with back to back data. This framework keeps up what is known as a memory (or a covered state) to adapt common conditions among data and yield progressions.

\section{BIOSIGNAL APPLICATION}

Analysis of ECG signal with determined frequencydomain measurements is an option for arrhythmia detection. In this strategy, frequency properties of the signal are examined (Electrophysiology 1996). 
The recurrence creation of the pulse can be spoken to in a diagram with power circulation versus frequency, i.e., powerspectral density (PSD), by which it is conceivable to pass judgment on the seriousness of recurrence parts in the scope of Very low recurrence (VLF: $<0.04 \mathrm{~Hz}$ ), low frequency (LF: $0.04-0.15 \mathrm{~Hz}$ ), and high frequency (HF: $0.15-0.4 \mathrm{~Hz}$ ). All out power in various groups and standardized absolute forces are utilized to assess the performance.

Old style techniques for spectral analysis are generally utilized for arrhythmia discovery in light of the simplicity of calculations utilized (much of the time, FFT), high preparing velocity, the unwavering quality of the examination results, and simplicity of usage with standard equipment units. The benefits of utilizing strategies, for example, FFT are their effortlessness and high computational velocities. Be that as it may, they endure from measurable flimsiness in the outcomes.

\section{CONCLUSION}

Biomedical sign and picture preparing care of includes various interests in the instructive and research field in biomedical structure. With the upgraded physiological information, a wide blend of creative works in clinical approachs utilizes this idea in the therapeutic applications. Further, prompted figurings dissected in the sign and picture preparing field typically wrapped up by time-rehash delineation approaches in the field of neurosciences, practical imaging and cardiovascular structure. In the impelled world, a part of the imaging modalities are as of now for the most part open, that can deal with crafted by disease and give definite information. The blend of electrophysiological sign and imaging techniques is a striking philosophy in the therapeutic field.

This precise survey gives a few settled calculations dependent on the combination of electrophysiological signal and practical imaging. The displayed contextual investigations presented a portrayal of ECG, EEG, EMG flag and imaging combination ventures because of the significance of this method in this specific situation. The future examination is looking at the performance of most exceptional calculations and techniques utilizing the combination of electrophysiological sign and utilitarian imaging.

\section{REFERENCES}

1. E. D. Übeyli and I. Güler, "Features extracted by eigenvector methods for detecting variability of EEG signals," Pattern Recognition Letters, vol. 28, no. 5, pp. 592-603, 2007.

2. S. A. Awang, M. Paulraj, and S. Yaacob, "Analysis of EEG signals by eigenvector methods," in Proceedings of the IEEE EMBS Conference on Biomedical Engineering and Sciences (IECBES '12), pp. 778-783, December 2012.

3. C. Guerrero-Mosquera and A. N. Vazquez, "New approach in features extraction for EEG signal detection," in Proceedings of the Annual International Conference of the IEEE Engineering in Medicine and Biology Society (EMBC '09), pp. 13-16, September 2009.

4. M. R. N. Kousarrizi, A. A. Ghanbari, M. Teshnehlab, M. Aliyari, and A. Gharaviri, "Feature extraction and classification of EEG signals using wavelet transform, SVM and artificial neural networks for brain computer interfaces," in Proceedings of the International Joint Conference on Bioinformatics, Systems Biology and Intelligent Computing (IJCBS '09), pp. 352-355, August 2009.

5. D. Cvetkovic, E. D. Übeyli, and I. Cosic, "Wavelet transform feature extraction from human PPG, ECG, and EEG signal responses to ELF
PEMF exposures: a pilot study," Digital Signal Processing, vol. 18, no. 5, pp. 861-874, 2008.

6. M. Baumert, A. Porta, and A. Cichocki, "Biomedical signal processing: From a conceptual framework to clinical applications [scanning the issue]," Proceedings of the IEEE, vol. 104, no. 2, pp. 220-222, 2016.

7. V. D. A. P. R. Rameshwari S Mane, A N Cheeran, "Cardiac arrhythmia detection by ecg feature extraction," International Journal of Engineering Research and Applications (IJERA), vol. 3, no. 2, pp. 327-332, 2013.

8. Gold, B., \& Morgan, N. (2000). Speech and audio signal processing : processing and perception of speech andmusic. New York: John Wiley. 